\title{
Error bounds associated with different versions of Hadamard inequalities of mid-point type
}

\author{
Muhammad Raees ${ }^{a, *}$, Matloob Anwar ${ }^{a}$, Ghulam Farid ${ }^{b}$ \\ a School of Natural Sciences, National University of Sciences and Technology, Islamabad, Pakistan. \\ ${ }^{b}$ Department of Mathematics, COMSATS University Islamabad, Attock Campus, Attock, Pakistan.
}

\begin{abstract}
In this paper, we establish the error bounds of different versions of mid-point type inequalities. At first, we prove two identities for fractional integrals involving the extended generalized Mittag-Leffler function and generalized exponential fractional integrals, and then we establish the corresponding error bound inequalities. Furthermore, we find a generalized inequality for error bound inequalities using a generalized identity. Also, we find some inequalities which formulate all error bound inequalities for various versions of Hadamard inequality. Finally, we present some examples of the central moment of a random variable.
\end{abstract}

Keywords: Convex function, extended generalized Mittag-Leffler function, generalized integral, Hadamard inequality.

2020 MSC: 26D07, 26D10, 26D15, 26A33.

(C)2021 All rights reserved.

\section{Preliminaries and introductory remarks}

Recall that a function $\mathrm{g}: \mathrm{K} \subset \mathbb{R} \rightarrow \mathbb{R}$ is called convex on $\mathrm{K}$, if the inequality

$$
g(r c+(1-r) d) \leqslant r g(c)+(1-r) g(d),
$$

holds for all $c, d \in K$ and $r \in[0,1]$.

The first fundamental inequality for convex functions is Hadamard inequality. The Hadamard inequality is one of the most fascinating inequality which can be stated as: If $g: K \subset \mathbb{R} \rightarrow \mathbb{R}$ is a convex function on $K$ and $c, d \in K$ with $c<d$, then

$$
g\left(\frac{c+d}{2}\right) \leqslant \frac{1}{d-c} \int_{c}^{d} g(x) d x \leqslant \frac{g(c)+g(d)}{2} .
$$

The Hadamard inequality due to its applications and geometric interpretation has attracted the attention of researchers. Due to which, various extensions of this inequality have been made by the researchers using innovative and new ideas, see $[8,9,14-16,18,20,21]$.

\footnotetext{
${ }^{*}$ Corresponding author

Email address: muhammad.raees@sns.nust.edu.pk, raeesqau1@gmail.com (Muhammad Raees)

doi: $10.22436 /$ jmcs.023.03.05
}

Received: 2020-04-02 Revised: 2020-05-25 Accepted: 2020-06-22 
The classical fractional integral operator is known as Riemann-Liouville fractional integrals. They are most studied operators due to their natural occurrence in half order differential equations. The RiemannLiouville fractional integral model of a function $\mathrm{g}$ of order $\gamma>0$ is given by [10, 19]:

$$
\gamma_{\mathrm{I}^{+}} \mathrm{g}(x)=\frac{1}{\Gamma(\gamma)} \int_{\mathrm{c}}^{x} \frac{\mathrm{g}(\tau)}{(\chi-\tau)^{1-\gamma}} \mathrm{d} \tau, \quad x>\mathrm{c} .
$$

The natural extension of Riemann-Liouville fractional integral (1.1) called generalized Riemann-Liouville fractional integral is given by $[10,19]$ :

$$
\underset{\mu}{\gamma} \mathrm{I}_{\mathrm{c}^{+}} \mathrm{g}(x)=\frac{1}{\Gamma(\gamma)} \int_{\mathcal{c}}^{x} \frac{\mu^{\prime}(\tau) g(\tau)}{(\mu(x)-\mu(\tau))^{1-\gamma}} \mathrm{d} \tau, \quad x>c .
$$

Kang et al. [9] generalized the integral (1.1) by adjoining an extended generalized Mittage-Leffler function as follows:

$$
w \Upsilon_{\beta, \gamma, v, c^{+}}^{\delta, r, s, c} g\left(x ; q^{*}\right)=\int_{c}^{x} \frac{E_{\beta, \gamma, v}^{\delta, r, s, c}\left(w(x-\tau)^{\beta} ; q^{*}\right) g(\tau)}{(x-\tau)^{1-\gamma}} d \tau, \quad x>c,
$$

where

$$
E_{\beta, \gamma, v}^{\delta, r, s, c}\left(u ; q^{*}\right)=\sum_{m=0}^{\infty} \frac{B_{q^{*}}(\delta+m s, c-\delta)(c)_{s m}}{B(\delta, c-\delta) \Gamma(\beta m+\gamma)(v)_{r m}} u^{m},
$$

is the extended generalized Mittag-leffler function with $B(u, v)$ is the well known Beta function and $B_{q^{*}}(u, v)$ is the generalized Beta function defined by

$$
\mathrm{B}_{\mathrm{q}^{*}}(\mathrm{u}, v)=\int_{0}^{1} \tau^{\mathrm{u}-1}(1-\tau)^{\nu-1} e^{\frac{-\mathrm{q}^{*}}{\tau(1-\tau)}} d \tau
$$

Recently, Farid [6] gave an integral formulation for various fractional integral operators as follows.

Definition 1.1. Let $g, \mu:[c, d] \rightarrow \mathbb{R}, 0<c<d$, be the functions such that $g$ be positive and $g \in L_{1}[c, d]$ and $\mu$ be differentiable and increasing. Also let $\rho$ be a positive function such that $\frac{\rho}{x}$ is increasing on $[c, \infty)$. Then for $x \in[c, d]$ the left-sided integral operators is defined by

$$
{ }_{\mu}^{\rho} F_{c^{+}} g(x)=\int_{c}^{x} \frac{\rho(\mu(x)-\mu(\tau)) g(\tau) \mu^{\prime}(\tau)}{\mu(x)-\mu(\tau)} d \tau, \quad x>c .
$$

It is worth mentioning that we can deduce different fractional integral operators by some suitable settings of functions $\rho$ and $\mu$.

(i) If $\rho(\mathfrak{u})=\frac{1}{\mathrm{k} \Gamma_{\mathrm{k}}(\gamma)} \mathbf{u}^{\frac{\gamma}{k}}$, then we have $\mathrm{k}$-analogue of generalized Riemann-Liouville fractional integral [13] as follows:

$$
{ }_{\mu}^{\gamma} \mathrm{I}_{\mathrm{c}^{+}}^{\mathrm{k}} \mathrm{g}(\mathrm{x})=\frac{1}{\mathrm{k} \Gamma_{\mathrm{k}}(\gamma)} \int_{\mathrm{c}}^{x} \frac{\mu^{\prime}(\tau) g(\tau)}{(\mu(x)-\mu(\tau))^{1-\frac{\gamma}{k}}} \mathrm{~d} \tau, x>c .
$$

For $k=1$, we get the generalized Riemann-Liouville fractional integral (1.2).

(ii) If we take $\rho(u)=\frac{u}{\gamma} \exp \left(-\frac{1-\gamma}{\gamma} u\right), \gamma \in(0,1)$, then we get the generalized exponential fractional integral [5] as following:

$$
\underset{\mu}{\gamma} \mathrm{E}_{\mathrm{c}^{+}} \mathrm{g}(\mathrm{x})=\frac{1}{\gamma} \int_{\mathrm{c}}^{x} \exp \left(-\frac{1-\gamma}{\gamma}(\mu(x)-\mu(\tau))\right) \mu^{\prime}(\tau) g(\tau) \mathrm{d} \tau, \quad x>c .
$$


(iii) If $\rho(u)=u^{\frac{\gamma}{k}} \mathcal{F}_{\rho, \gamma}^{\sigma, k}\left(w(u)^{\rho}\right)$ in (1.4), then we obtain the generalized k-fractional integral operator [22] as follows:

$$
{ }_{\rho}^{\sigma} \zeta_{\gamma, c^{+} ; w}^{k, \mu} g(x)=\int_{c}^{x} \frac{\mu^{\prime}(\tau) \mathcal{F}_{\rho, \gamma}^{\sigma, k}\left(w(\mu(x)-\mu(\tau))^{\rho}\right) g(\tau)}{(\mu(x)-\mu(\tau))^{1-\frac{\gamma}{k}}} d \tau, \quad x>c,
$$

where

$$
\mathcal{F}_{\rho, \gamma}^{\sigma, k}(x):=\sum_{n=0}^{\infty} \frac{\sigma(n)}{k \Gamma_{k}(\rho k n+\gamma)} x^{n}, \quad(\rho, \gamma>0 ;|x|<R) \text { with } R>0,
$$

and the coefficients $\sigma(n)(n \in \mathbb{N} \cup\{0\})$ form a bounded sequence of positive real numbers.

(iv) If we choose $\rho(u)=u^{\gamma} E_{\beta, \gamma, v}^{\delta, r, s, c}\left(w(u)^{\beta} ; q^{*}\right)$, then we get a natural extension of the fractional integral operator (1.3) as follows:

$$
\underset{\mu}{w} \Upsilon_{\beta, \gamma, v, c^{+}}^{\delta, r, s, c} g(x)=\int_{c}^{x} \frac{\mu^{\prime}(\tau) E_{\beta, \gamma, v}^{\delta, r, s, c}\left(w(\mu(x)-\mu(\tau))^{\beta} ; q^{*}\right) g(\tau)}{(\mu(x)-\mu(\tau))^{1-\gamma}} d \tau, \quad x>c .
$$

Some more operators can be deduced from (1.4). For a detailed study of fractional and conformable integral operators which can be obtained from (1.4), we refer to [7].

Kang et al. [9] established a generalized version of Hadamard inequality utilizing operator (1.3).

Theorem 1.2. Let $\mathrm{g}:[\mathrm{c}, \mathrm{d}] \rightarrow \mathbb{R}$ be a positive function with $0 \leqslant \mathrm{c}<\mathrm{d}$ and $\mathrm{g} \in \mathrm{L}_{1}[\mathrm{c}, \mathrm{d}]$. If $\mathrm{g}$ is a convex function on $[\mathrm{c}, \mathrm{d}]$, then the following inequality for the fractional integral (1.3) holds:

$$
\begin{aligned}
g\left(\frac{c+d}{2}\right) w^{\prime} \Upsilon_{\beta, \gamma, v, c^{+}}^{\delta, r, s, c} 1\left(d ; q^{*}\right) & \leqslant \frac{w^{\prime} \Upsilon_{\beta, \gamma, v, c^{+}}^{\delta, r, s, c} g\left(d ; q^{*}\right)+{ }^{w^{\prime}} \Upsilon_{\beta, \gamma, v, d^{-}}^{\delta, r, s, c} g\left(c ; q^{*}\right)}{2} \\
& \leqslant \frac{g(c)+g(d)}{2}{ }^{w^{\prime}} \Upsilon_{\beta, \gamma, v, p^{+}}^{\delta, r, s, c} 1\left(c ; q^{*}\right),
\end{aligned}
$$

where $w^{\prime}=\frac{w}{(\mathrm{~d}-\mathrm{c})^{\gamma}}$.

Set et al. [21] introduced a generalized version of Hadamard inequality via fractional integral operator (1.6).

Theorem 1.3. Let $k, \rho, \gamma \in \mathbb{R}^{+}, w \in \mathbb{R}_{0}^{+}$and $\sigma(\mathrm{m}) \in \mathbb{R}^{+}\left(\mathrm{m} \in \mathbb{N}_{0}\right)$ be a bounded sequence. Also, let $\mu:[\mathrm{c}, \mathrm{d}] \rightarrow$ $\mathbb{R}$ be a an increasing function on $[\mathrm{c}, \mathrm{d}]$ having continuous derivative $\mu^{\prime}(\mathrm{x})$ on $(\mathrm{c}, \mathrm{d})$. If $\mathrm{g}$ is a convex function on [c, d], then following Hermite-Hadamard type inequalities for generalized k-fractional integral (1.6) hold:

$$
g\left(\frac{c+d}{2}\right) \leqslant \frac{1}{2 k}\left[{ }_{\rho}^{\sigma}{\zeta^{k, \mu}, \frac{c+d}{2}+;}^{k} G(d)+{ }_{\rho}^{\sigma} \zeta_{\gamma, \frac{c+d}{2}}^{k, \mu} ; w(c)\right] \leqslant \frac{g(c)+g(d)}{2}
$$

where $G(x)=g(x)+g(c+d-x)$.

Motivated by these papers, we present the following work whose purpose is to develop some generalized error bound inequalities for the aforementioned fractional integrals. We have organized the paper as follows.

In Section 2, we prove two identities for fractional integral operators (1.5) and (1.7) and establish corresponding error bound inequalities. In Section 3, we develop some generalized error bound inequalities via generalized integral operator (1.4). In Section 4, we construct some new inequalities for central moment of the random variable. In Section 5, we conclude our results. Through out this paper, we consider $G(x)=g(x)+\widetilde{g}(x)$, where $g$ is a convex function and $\widetilde{g}(x)=g(c+d-x)$ for all $x \in[c, d]$. 


\section{Some fractional Hadamard inequalities at midpoint}

In this section, we give some error estimates for Hadamard inequalities associated to the fractional integral operators (1.5) and (1.6). We start with the following identity.

Lemma 2.1. Let $\mu:[c, d] \rightarrow \mathbb{R}$ be a positive monotone increasing function with continuous derivative $\mu^{\prime}$ on $(c, d)$. Let $\mathrm{g}:[\mathrm{c}, \mathrm{d}] \rightarrow \mathbb{R}$ be a differentiable function on $(\mathrm{c}, \mathrm{d})$ with $0 \leqslant \mathrm{c}<\mathrm{d}$. If $\mathrm{g}^{\prime} \in \mathrm{L}_{1}[\mathrm{c}, \mathrm{d}]$, then the following identity holds for operator (1.7):

$$
\begin{gathered}
{ }^{\mu} R_{\beta, \gamma, \gamma}^{\delta, r, s, c}(1) g\left(\frac{c+d}{2}\right)-\frac{1}{d-c}\left[\underset{\mu}{w} \Upsilon_{\beta, \gamma, v, \frac{c+d}{2}}^{\delta, r, s, c} G(d)+{ }_{\mu}^{w} \Upsilon_{\beta, \gamma, v, \frac{c+d}{2}}^{\delta, r, s, c} G(c)\right] \\
=\frac{d-c}{4} \int_{0}^{1} R_{\beta, \gamma, v}^{\delta, r, s, c}(r)\left[g^{\prime}\left(\frac{r}{2} d+\frac{2-r}{2} c\right)-g^{\prime}\left(\frac{r}{2} c+\frac{2-r}{2} d\right)\right] d r
\end{gathered}
$$

where

$$
\begin{aligned}
{ }^{\mu} R_{\beta, \gamma, v}^{\delta, r, s, c}(r)= & {\left[\mu(d)-\mu\left(\frac{r}{2} c+\frac{2-r}{2} d\right)\right]^{\gamma} E_{\beta, \gamma+1, v}^{\delta, r, s, c}\left(\left[\mu(d)-\mu\left(\frac{r}{2} c+\frac{2-r}{2} d\right)\right]^{\beta} ; q^{*}\right) } \\
& +\left[\mu\left(\frac{r}{2} d+\frac{2-r}{2} c\right)-\mu(c)\right]^{\gamma} E_{\beta, \gamma+1, v}^{\delta, r, s, c}\left(\left[\mu\left(\frac{r}{2} d+\frac{2-r}{2} c\right)-\mu(c)\right]^{\beta} ; q^{*}\right) .
\end{aligned}
$$

Proof. We have

$$
\begin{aligned}
& \int_{0}^{1} \mu R_{\beta, \gamma, v}^{\delta, r, s, c}(r)\left[g^{\prime}\left(\frac{r}{2} d+\frac{2-r}{2} c\right)-g^{\prime}\left(\frac{r}{2} c+\frac{2-r}{2} d\right)\right] d r \\
& \quad=\int_{0}^{1} \mu R_{\beta, \gamma, v}^{\delta, r, s, c}(r) g^{\prime}\left(\frac{r}{2} d+\frac{2-r}{2} c\right) d r-\int_{0}^{1} \mu R_{\beta, \gamma, v}^{\delta, r, s, c}(r) g^{\prime}\left(\frac{r}{2} c+\frac{2-r}{2} d\right) d r .
\end{aligned}
$$

Integrating by parts, we have

$$
\begin{aligned}
& \int_{0}^{1} \mu R_{\beta, \gamma, v}^{\delta, r, s, c}(r) g^{\prime}\left(\frac{r}{2} d+\frac{2-r}{2} c\right) d r=\left.\frac{2}{d-c}{ }^{\mu} R_{\beta, \gamma, v}^{\delta, r, s, c}(r) g\left(\frac{r}{2} d+\frac{2-r}{2} c\right)\right|_{0} ^{1} \\
& -\frac{2}{d-c} \sum_{m=0}^{\infty} \frac{B_{q^{*}}(\delta+m s, c-\delta)(c)_{s m}}{B(\delta, c-\delta) \Gamma(\beta m+\gamma)(v)_{r m}} \int_{0}^{1} \frac{\mu^{\prime}\left(\frac{r}{2} c+\frac{2-r}{2} d\right) g\left(\frac{r}{2} d+\frac{2-r}{2} c\right)}{\left[\mu(d)-\mu\left(\frac{r}{2} c+\frac{2-r}{2} d\right)\right]^{1-\gamma-\beta m} d r} \\
& -\frac{2}{d-c} \sum_{m=0}^{\infty} \frac{B_{q^{*}}(\delta+m s, c-\delta)(c)_{s m}}{B(\delta, c-\delta) \Gamma(\beta m+\gamma)(v)_{r m}} \int_{0}^{1} \frac{\mu^{\prime}\left(\frac{r}{2} d+\frac{2-r}{2} c\right) g\left(\frac{r}{2} d+\frac{2-r}{2} c\right)}{\left[\mu\left(\frac{r}{2} d+\frac{2-r}{2} c\right)-\mu(c)\right]^{1-\gamma-\beta m}} d r \\
& =\frac{2}{d-c}{ }^{\mu} R_{\beta, \gamma, v}^{\delta, r, s, c}(1) g\left(\frac{c+d}{2}\right) \\
& -\frac{2}{d-c} \int_{0}^{1} \frac{\mu^{\prime}\left(\frac{r}{2} c+\frac{2-r}{2} d\right) E_{\beta, \gamma, v}^{\delta, r, s, c}\left(\left[\mu(d)-\mu\left(\frac{r}{2} c+\frac{2-r}{2} d\right)\right]^{\gamma} ; q^{*}\right) g\left(\frac{r}{2} d+\frac{2-r}{2} c\right)}{\left[\mu(d)-\mu\left(\frac{r}{2} c+\frac{2-r}{2} d\right)\right]^{1-\gamma}} d r \\
& -\frac{2}{d-c} \int_{0}^{1} \frac{\mu^{\prime}\left(\frac{r}{2} d+\frac{2-r}{2} c\right) E_{\beta, \gamma, v}^{\delta, r, s, c}\left(\left[\mu\left(\frac{r}{2} d+\frac{2-r}{2} c\right)-\mu(c)\right]^{\gamma} ; q^{*}\right) g\left(\frac{r}{2} d+\frac{2-r}{2} c\right)}{\left[\mu\left(\frac{r}{2} d+\frac{2-r}{2} c\right)-\mu(c)\right]^{1-\gamma}} d r .
\end{aligned}
$$


Similarly,

$$
\begin{aligned}
& \int_{0}^{1} \mu R_{\beta, \gamma, v}^{\delta, r, s, c}(r) g^{\prime}\left(\frac{r}{2} c+\frac{2-r}{2} d\right) d r=-\frac{2}{d-c}{ }^{\mu} R_{\beta, \gamma, v}^{\delta, r, s, c}(1) g\left(\frac{c+d}{2}\right) \\
& +\frac{2}{d-c} \int_{0}^{1} \frac{\mu^{\prime}\left(\frac{r}{2} c+\frac{2-r}{2} d\right) E_{\beta, \gamma, v}^{\delta, r, s, c}\left(\left[\mu(d)-\mu\left(\frac{r}{2} c+\frac{2-r}{2} d\right)\right]^{\gamma} ; q^{*}\right) g\left(\frac{r}{2} c+\frac{2-r}{2} d\right)}{\left[\mu(d)-\mu\left(\frac{r}{2} c+\frac{2-r}{2} d\right)\right]^{1-\gamma}} d r \\
& +\frac{2}{d-c} \int_{0}^{1} \frac{\mu^{\prime}\left(\frac{r}{2} d+\frac{2-r}{2} c\right) E_{\beta, \gamma, v}^{\delta, r, s, c}\left(\left[\mu\left(\frac{r}{2} d+\frac{2-r}{2} c\right)-\mu(c)\right]^{\gamma} ; q^{*}\right) g\left(\frac{r}{2} c+\frac{2-r}{2} d\right)}{\left[\mu\left(\frac{r}{2} d+\frac{2-r}{2} c\right)-\mu(c)\right]^{1-\gamma}} d r .
\end{aligned}
$$

Applying (1.7) in (2.1) and (2.2), we respectively have

$$
\begin{aligned}
\int_{0}^{1} \mu R_{\beta, \gamma, v}^{\delta, r, s, c}(r) g^{\prime}\left(\frac{r}{2} d+\frac{2-r}{2} c\right) d r= & \frac{2}{d-c} \mu R_{\beta, \gamma, v}^{\delta, r, s, c}(1) g\left(\frac{c+d}{2}\right)-\frac{4}{(d-c)^{2}}{ }^{w} \Upsilon_{\beta, \gamma, v, \frac{c+d}{2}}^{\delta, r, s, c}+g(d) \\
& -\frac{4}{(d-c)^{2}} \stackrel{w}{\mu} \Upsilon_{\beta, \gamma, \gamma, \frac{c+d}{2}}^{\delta, r, s, c}-g(c) .
\end{aligned}
$$

and

$$
\begin{aligned}
& \int_{0}^{1} \mu R_{\beta, \gamma, v}^{\delta, r, s, c}(r) g^{\prime}\left(\frac{r}{2} c+\frac{2-r}{2} d\right) d r=-\frac{2}{d-c}{ }^{\mu} R_{\beta, \gamma, v}^{\delta, r, s, c}(1) g\left(\frac{c+d}{2}\right)+\frac{4}{(d-c)^{2}} \mu^{w} \Upsilon_{\beta, \gamma, v, \frac{c+d}{2}}^{\delta, r, s, c} g(d) \\
& -\frac{4}{(d-c)^{2}} \stackrel{w}{\mu} \Upsilon_{\beta, \gamma, v, \frac{c+d}{2}}^{\delta, r, s, c}-\widetilde{g}(c) .
\end{aligned}
$$

Equations (2.3) and (2.4) give the required identity.

Lemma 2.2. Let $\mu:[c, d] \rightarrow \mathbb{R}$ be a positive and monotone increasing function with continuous derivative $\mu^{\prime}$ on $(\mathrm{c}, \mathrm{d})$. Let $\mathrm{g}:[\mathrm{c}, \mathrm{d}] \rightarrow \mathbb{R}$ be a differentiable function on $(\mathrm{c}, \mathrm{d})$ with $0 \leqslant \mathrm{c}<\mathrm{d}$. If $\mathrm{g}^{\prime} \in \mathrm{L}_{1}[\mathrm{c}, \mathrm{d}]$, then the following identity holds for the fractional integral operator (1.5):

$$
\begin{aligned}
& N_{\gamma}^{\mu}(1) g\left(\frac{c+d}{2}\right)-\frac{1-\gamma}{2}\left[\gamma_{\mu} E_{\frac{c+d}{2}} G(c)+{ }_{\mu}^{\gamma} E_{\frac{c+d}{2}} G(d)\right] \\
& =\frac{d-c}{4} \int_{0}^{1} N_{\gamma}^{\mu}(r)\left[g^{\prime}\left(\frac{r}{2} d+\frac{2-r}{2} c\right)-g^{\prime}\left(\frac{r}{2} c+\frac{2-r}{2} d\right)\right] d r
\end{aligned}
$$

where

$$
N_{\gamma}^{\mu}(r):=2-\exp \left(-A\left(\left(\mu(d)-\mu\left(\frac{r}{2} c+\frac{2-r}{2} d\right)\right)\right)\right)-\exp \left(-A\left(\left(\mu\left(\frac{r}{2} d+\frac{2-r}{2} c\right)-\mu(c)\right)\right)\right),
$$

and $\mathrm{A}=\frac{1-\gamma}{\gamma}, \gamma \in(0,1)$.

Proof. Integrating by parts, we have

$$
\int_{0}^{1} N_{\gamma}^{\mu}(r) g^{\prime}\left(\frac{r}{2} d+\frac{2-r}{2} c\right) d r
$$




$$
\begin{aligned}
= & \left.\frac{2}{d-c} N_{\gamma}^{\mu}(r) g\left(\frac{r}{2} d+\frac{2-r}{2} c\right)\right|_{0} ^{1} \\
& -A \int_{0}^{1} \exp \left(-A\left(\mu(d)-\mu\left(\frac{r}{2} c+\frac{2-r}{2} d\right)\right)\right) \mu^{\prime}\left(\frac{r}{2} c+\frac{2-r}{2} d\right) g\left(\frac{r}{2} d+\frac{2-r}{2} c\right) d r \\
& -A \int_{0}^{1} \exp \left(-A\left(\mu\left(\frac{r}{2} d+\frac{2-r}{2} c\right)-\mu(c)\right)\right) \mu^{\prime}\left(\frac{r}{2} d+\frac{2-r}{2} c\right) g\left(\frac{r}{2} d+\frac{2-r}{2} c\right) d r \\
= & \frac{2}{d-c} N_{\gamma}^{\mu}(1) g\left(\frac{c+d}{2}\right)-\frac{2 A}{d-c} \int_{\frac{c+d}{2}}^{d} \exp (-A(\mu(d)-\mu(x))) \mu^{\prime}(x) \widetilde{g}(x) d x \\
& -\frac{2 A}{d-c} \int_{c}^{\frac{c+d}{2}} \exp (-A(\mu(x)-\mu(c))) \mu^{\prime}(x) g(x) d x .
\end{aligned}
$$

Similarly,

$$
\begin{aligned}
\int_{0}^{1} N_{\gamma}^{\mu}(r) g^{\prime}\left(\frac{r}{2} c+\frac{2-r}{2} d\right) d r= & -\frac{2}{(d-c)} N_{\gamma}^{\mu}(1) g\left(\frac{c+d}{2}\right) \\
& +\frac{2 A}{d-c} \int_{\frac{c+d}{2}}^{d} \exp (-A(\mu(d)-\mu(x))) \mu^{\prime}(x) g(x) d x \\
& +\frac{2 A}{d-c} \int_{c}^{\frac{c+d}{2}} \exp (-A(\mu(x)-\mu(c))) \mu^{\prime}(x) \widetilde{g}(x) d x .
\end{aligned}
$$

By applying (1.5) to (2.5) and (2.6), we have

$$
\begin{aligned}
\int_{0}^{1} N_{\gamma}^{\mu}(r) g^{\prime}\left(\frac{r}{2} d+\frac{2-r}{2} c\right) d r= & \frac{2}{(d-c)} N_{\gamma}^{\mu}(1) g\left(\frac{c+d}{2}\right)-\frac{2(1-\gamma)}{d-c} \gamma_{\mu} E_{\frac{c+d}{2}}^{\gamma} \widetilde{g}(d) \\
& -\frac{2(1-\gamma)}{d-c} \gamma_{\mu} E_{\frac{c+d}{2}}-g(c),
\end{aligned}
$$

and

$$
\begin{aligned}
\int_{0}^{1} N_{\gamma}^{\mu}(r) g^{\prime}\left(\frac{r}{2} c+\frac{2-r}{2} d\right) d r= & -\frac{2}{(d-c)} N_{\gamma}^{\mu}(1) g\left(\frac{c+d}{2}\right)+\frac{2(1-\gamma)}{d-c} \gamma_{\mu} E_{\frac{c+d}{2}}+g(d) \\
& +\frac{2(1-\gamma)}{d-c} \gamma_{\mu} E_{\frac{c+d}{2}}-\widetilde{g}(c) .
\end{aligned}
$$

Equations (2.7) and (2.8) lead to the required identity.

Now, we give some Hadamard inequalities for fractional integrals (1.7) and (1.5).

Theorem 2.3. Let $\mu:[c, d] \rightarrow \mathbb{R}$ be a positive monotone increasing function with continuous derivative $\mu^{\prime}$ on $(\mathrm{c}, \mathrm{d})$. Let $\mathrm{g}:[\mathrm{c}, \mathrm{d}] \rightarrow \mathbb{R}$ be a differentiable function on $(\mathrm{c}, \mathrm{d})$ with $0 \leqslant \mathrm{c}<\mathrm{d}$ such that $\mathrm{g}^{\prime} \in \mathrm{L}_{1}[\mathrm{c}, \mathrm{d}]$. If $\left|\mathrm{g}^{\prime}\right|$ is 
convex, then the following inequality holds for the fractional integral (1.7):

$$
\begin{aligned}
& \left|{ }^{\mu} R_{\beta, \gamma, v}^{\delta, r, s, c}(1) g\left(\frac{c+d}{2}\right)-\frac{1}{d-c}\left[\underset{\mu}{w} \Upsilon_{\beta, \gamma, \gamma, \frac{c+d}{2}}^{\delta, r, s, c} G(d)+\mu_{\mu}^{w} \Upsilon_{\beta, \gamma, v, \frac{c+d}{2}}^{\delta, r, s, c}-G(c)\right]\right| \\
& \quad \leqslant \frac{(d-c)\left[\left|g^{\prime}(c)\right|+\left|g^{\prime}(d)\right|\right]}{4} \int_{0}^{1}\left|\mu R_{\beta, \gamma, v}^{\delta, r, s, c}(r)\right| d r .
\end{aligned}
$$

Proof. By Lemma 2.1, and property of modulus, we have

$$
\begin{gathered}
\left|\mu R_{\beta, \gamma, v}^{\delta, r, s, c}(1) g\left(\frac{c+d}{2}\right)-\frac{1}{d-c}\left[{ }_{\mu}^{w} \Upsilon_{\beta, \gamma, v, \frac{c+d}{2}}^{\delta, r, s, c} G(d)+{ }_{\mu}^{w} \Upsilon_{\beta, \gamma, v, \frac{c+d}{2}}^{\delta, r, s, c} G(c)\right]\right| \\
\leqslant \frac{(d-c)}{4} \int_{0}^{1}\left|\mu R_{\beta, \gamma, v}^{\delta, r, s, c}(r)\right|\left[\left|g^{\prime}\left(\frac{r}{2} d+\frac{2-r}{2} c\right)\right|+\left|g^{\prime}\left(\frac{r}{2} c+\frac{2-r}{2} d\right)\right|\right] d r .
\end{gathered}
$$

By convexity of $\left|g^{\prime}\right|$, we get

$$
\begin{aligned}
& \left|g^{\prime}\left(\frac{r}{2} d+\frac{2-r}{2} c\right)\right|+\left|g^{\prime}\left(\frac{r}{2} c+\frac{2-r}{2} d\right)\right| \\
& \leqslant \frac{r}{2}\left|g^{\prime}(d)\right|+\frac{2-r}{2}\left|g^{\prime}(c)\right|+\frac{r}{2}\left|g^{\prime}(c)\right|+\frac{2-r}{2}\left|g^{\prime}(d)\right|=\left|g^{\prime}(c)\right|+\left|g^{\prime}(d)\right| .
\end{aligned}
$$

Using (2.10) in (2.9), we obtain the required inequality.

Theorem 2.4. Let $\mu:[c, d] \rightarrow \mathbb{R}$ be a positive and monotone increasing function with continuous derivative $\mu^{\prime}$ on $(\mathrm{c}, \mathrm{d})$. Let $\mathrm{g}:[\mathrm{c}, \mathrm{d}] \rightarrow \mathbb{R}$ be a differentiable function on $(\mathrm{c}, \mathrm{d})$ with $0 \leqslant \mathrm{c}<\mathrm{d}$ such that $\mathrm{g}^{\prime} \in \mathrm{L}_{1}[\mathrm{c}, \mathrm{d}]$. If $\left|\mathrm{g}^{\prime}\right|$ is convex, then the following inequality holds for the fractional integral operator 1.5:

$$
\begin{aligned}
& \left|N_{\gamma}^{\mu}(1) g\left(\frac{c+d}{2}\right)-\frac{1-\gamma}{2}\left[\underset{\mu}{\gamma} E_{\frac{c+d}{2}}-G(c)+{ }_{\mu}^{\gamma} E_{\frac{c+d}{2}} G(d)\right]\right| \\
& \leqslant \frac{(d-c)\left[\left|g^{\prime}(c)\right|+\left|g^{\prime}(d)\right|\right]}{4} \int_{0}^{1}\left|N_{\gamma}^{\mu}(r)\right| d r .
\end{aligned}
$$

Proof. By Lemma 2.2 and following the steps in the proof of Theorem 2.3, we obtain the required inequality.

Theorem 2.5. Let $\mu:[c, d] \rightarrow \mathbb{R}$ be a positive monotone increasing function with continuous derivative $\mu^{\prime}$ on $(\mathrm{c}, \mathrm{d})$. Let $\mathrm{g}:[\mathrm{c}, \mathrm{d}] \rightarrow \mathbb{R}$ be a differentiable function on $(\mathrm{c}, \mathrm{d})$ with $0 \leqslant \mathrm{c}<\mathrm{d}$ such that $\mathrm{g}^{\prime} \in \mathrm{L}_{1}[\mathrm{c}, \mathrm{d}]$. If $\left|g^{\prime}\right|^{q}, q>1$ is convex, then the following inequality holds for the fractional integral (1.7):

$$
\begin{aligned}
& \left|\mu_{R_{\beta, \gamma, v}^{\delta, r, s, c}}(1) g\left(\frac{c+d}{2}\right)-\frac{1}{d-c}\left[\underset{\mu}{w} \Upsilon_{\beta, \gamma, v, \frac{c+d}{2}}^{\delta, r, s, c} G(d)+\underset{\mu}{w} \Upsilon_{\beta, \gamma, v, \frac{c+d}{2}}^{\delta, r, s, c}-G(c)\right]\right| \\
& \quad \leqslant \frac{(d-c)}{4}\left(\int_{0}^{1}\left|\mu R_{\beta, \gamma, v}^{\delta, r, s, c}(r)\right|^{p} d r\right)^{\frac{1}{p}}\left\{\left[\frac{\left|g^{\prime}(d)\right|^{q}+3\left|g^{\prime}(c)\right|^{q}}{4}\right]^{\frac{1}{q}}+\left[\frac{\left|g^{\prime}(c)\right| q+3\left|g^{\prime}(d)\right|^{q}}{4}\right]^{\frac{1}{q}}\right\},
\end{aligned}
$$

where $\frac{1}{p}+\frac{1}{q}=1, q>1$. 
Proof. From Lemma 2.1, we have

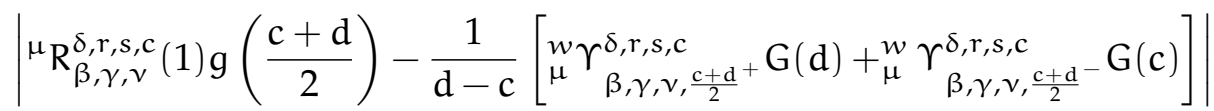

$$
\begin{aligned}
& \leqslant \frac{(d-c)}{4}\left\{\int_{0}^{1}\left|\mu R_{\beta, \gamma, v}^{\delta, r, s, c}(r)\right|\left|g^{\prime}\left(\frac{r}{2} d+\frac{2-r}{2} c\right)\right| d r+\int_{0}^{1}\left|\mu R_{\beta, \gamma, v}^{\delta, r, s, c}(r)\right|\left|g^{\prime}\left(\frac{r}{2} c+\frac{2-r}{2} d\right)\right| d r\right\} .
\end{aligned}
$$

Now by the Hölder's inequality, we have

$$
\int_{0}^{1}\left|{ }^{\mu} R_{\beta, \gamma, v}^{\delta, r, s, c}(r)\right|\left|g^{\prime}\left(\frac{r}{2} d+\frac{2-r}{2} c\right)\right| d r \leqslant\left(\int_{0}^{1}\left|\mu R_{\beta, \gamma, v}^{\delta, r, s, c}(r)\right|^{p} d r\right)^{\frac{1}{p}}\left(\int_{0}^{1}\left|g^{\prime}\left(\frac{r}{2} d+\frac{2-r}{2} c\right)\right|^{q} d r\right)^{\frac{1}{q}},
$$

and

$$
\int_{0}^{1}\left|\mu R_{\beta, \gamma, v}^{\delta, r, s, c}(r)\right|\left|g^{\prime}\left(\frac{r}{2} c+\frac{2-r}{2} d\right)\right| d r \leqslant\left(\int_{0}^{1}\left|\mu R_{\beta, \gamma, v}^{\delta, r, s, c}(r)\right|^{p} d r\right)^{\frac{1}{p}}\left(\int_{0}^{1}\left|g^{\prime}\left(\frac{r}{2} c+\frac{2-r}{2} d\right)\right|^{q} d r\right)^{\frac{1}{q}} .
$$

Since $\left|g^{\prime}\right|^{q}, q>1$ is convex, so we have

$$
\int_{0}^{1}\left|g^{\prime}\left(\frac{r}{2} d+\left(\frac{2-r}{2}\right) c\right)\right|^{q} d r \leqslant \frac{\left|g^{\prime}(d)\right|^{q}+3\left|g^{\prime}(c)\right|^{q}}{4}
$$

and

$$
\int_{0}^{1}\left|g^{\prime}\left(\frac{r}{2} c+\left(\frac{2-r}{2}\right) d\right)\right|^{q} d r \leqslant \frac{\left|g^{\prime}(c)\right|^{q}+3\left|g^{\prime}(d)\right|^{q}}{4} .
$$

Utilizing (2.12), (2.13), (2.14), and (2.15) in inequality (2.11), we obtain the desired inequality.

Theorem 2.6. Let $\mu:[c, d] \rightarrow \mathbb{R}$ be a positive and monotone increasing function with continuous derivative $\mu^{\prime}$ on $(\mathrm{c}, \mathrm{d})$. Let $\mathrm{g}:[\mathrm{c}, \mathrm{d}] \rightarrow \mathbb{R}$ be a differentiable function on $(\mathrm{c}, \mathrm{d})$ with $0 \leqslant \mathrm{c}<\mathrm{d}$ such that $\mathrm{g}^{\prime} \in \mathrm{L}_{1}[\mathrm{c}, \mathrm{d}]$. If $\left|g^{\prime}\right|^{\mathrm{q}}, \mathrm{q}>1$ is convex, then the following inequality holds for the fractional integral operator (1.5):

$$
\begin{aligned}
& \left|N_{\gamma}^{\mu}(1) g\left(\frac{c+d}{2}\right)-\frac{1-\gamma}{2}\left[\underset{\mu}{\gamma} E_{\frac{c+d}{2}}-G(c)+{ }_{\mu}^{\gamma} E_{\frac{c+d}{2}}+G(d)\right]\right| \\
& \leqslant \frac{(d-c)}{4}\left(\int_{0}^{1}\left|N_{\gamma}^{\mu}(r)\right|^{p} d r\right)^{\frac{1}{p}}\left\{\left[\frac{\left|g^{\prime}(d)\right|^{q}+3\left|g^{\prime}(c)\right|^{q}}{4}\right]^{\frac{1}{q}}+\left[\frac{\left|g^{\prime}(c)\right|^{q}+3\left|g^{\prime}(d)\right|^{q}}{4}\right]^{\frac{1}{q}}\right\},
\end{aligned}
$$

where $\frac{1}{\mathrm{p}}+\frac{1}{\mathrm{q}}=1, \mathrm{q}>1$.

Proof. The required inequality is obtained by Lemma 2.2 and application of the Hölder's inequality.

\section{Generalized integral identity}

Now and onward we consider,

$$
\Delta_{\rho, \mu}(r)=\int_{0}^{r} \omega_{\rho, \mu}(s) d r
$$


where

$$
\omega_{\rho, \mu}(s)=\frac{\rho\left(\mu\left(\frac{s}{2} d+\frac{2-s}{2} c\right)-\mu(c)\right)}{\mu\left(\frac{s}{2} d+\frac{2-s}{2} c\right)-\mu(c)} \mu^{\prime}\left(\frac{s}{2} d+\frac{2-s}{2} c\right)+\frac{\rho\left(\mu(d)-\mu\left(\frac{s}{2} c+\frac{2-s}{2} d\right)\right)}{\mu(d)-\mu\left(\frac{s}{2} c+\frac{2-s}{2} d\right)} \mu^{\prime}\left(\frac{s}{2} c+\frac{2-s}{2} d\right) .
$$

Lemma 3.1. Let $\mathrm{g}, \mu:[\mathrm{c}, \mathrm{d}] \rightarrow \mathbb{R}, 0<\mathrm{c}<\mathrm{d}$, be the functions such that $\mathrm{g}$ be a differentiable and positive with $\mathrm{g} \in \mathrm{L}_{1}[\mathrm{c}, \mathrm{d}]$ and $\mu$ be differentiable and increasing. Also let $\rho$ be a positive function such that $\frac{\rho}{\mathrm{x}}$ is increasing on $[\mathrm{c}, \infty)$. If $\mathrm{g}^{\prime} \in \mathrm{L}_{1}[\mathrm{c}, \mathrm{d}]$, then the following identity holds for the generalized integral operator (1.4):

$$
\begin{aligned}
& \Delta_{\rho, \mu}(1) g\left(\frac{c+d}{2}\right)-\frac{1}{d-c}\left[\rho_{\mu} F_{\left(\frac{c+d}{2}\right)^{+}} G(d)+{ }_{\mu}^{\rho} F_{\left(\frac{c+d}{2}\right)^{-}} G(d)\right] \\
& =\frac{d-c}{4} \int_{0}^{1} \Delta_{\rho, \mu}(r)\left[g^{\prime}\left(\frac{r}{2} d+\frac{2-r}{2} c\right)-g^{\prime}\left(\frac{r}{2} c+\frac{2-r}{2} d\right)\right] d r .
\end{aligned}
$$

Proof. Here,

$$
\begin{aligned}
& \int_{0}^{1} \Delta_{\rho, \mu}(r)\left[g^{\prime}\left(\frac{r}{2} d+\frac{2-r}{2} c\right)-g^{\prime}\left(\frac{r}{2} c+\frac{2-r}{2} d\right)\right] d r \\
& \quad=\int_{0}^{1} \Delta_{\rho, \mu}(\xi) g^{\prime}\left(\frac{r}{2} d+\frac{2-r}{2} c\right) d r-\int_{0}^{1} \Delta_{\rho, \mu}(\xi) g^{\prime}\left(\frac{r}{2} c+\frac{2-r}{2} d\right) d r .
\end{aligned}
$$

By integrating by parts, we have

$$
\begin{aligned}
\int_{0}^{1} \Delta_{\rho, \mu}(r) g^{\prime}\left(\frac{r}{2} d+\frac{2-r}{2} c\right) d r= & \left.\frac{2}{d-c} \Delta_{\rho, \mu}(r) g\left(\frac{r}{2} d+\frac{2-r}{2} c\right)\right|_{0} ^{1} \\
& -\frac{2}{d-c} \int_{0}^{1} \omega_{\rho, \mu}(r) g\left(\frac{r}{2} d+\frac{2-r}{2} c\right) d r \\
= & \frac{2}{d-c} \Delta_{\rho, \mu}(1) g\left(\frac{c+d}{2}\right)-\frac{4}{(d-c)^{2}} \int_{c}^{\frac{c+d}{2}} \frac{\rho(\mu(x)-\mu(c)) \mu^{\prime}(x) g(x)}{\mu(x)-\mu(c)} d x \\
& -\frac{4}{(d-c)^{2}} \int_{\frac{c+d}{2}}^{d} \frac{\rho(\mu(d)-\mu(x)) \mu^{\prime}(x) \widetilde{g}(x)}{\mu(d)-\mu(x)} d x .
\end{aligned}
$$

Similarly,

$$
\begin{aligned}
\int_{0}^{1} \Delta_{\rho, \mu}(r) g^{\prime}\left(\frac{r}{2} c+\frac{2-r}{2} d\right) d r= & -\frac{2}{d-c} \Delta_{\rho, \mu}(1) g\left(\frac{c+d}{2}\right) \\
& +\frac{4}{(d-c)^{2}} \int_{c}^{\frac{c+d}{2}} \frac{\rho(\mu(x)-\mu(c)) \mu^{\prime}(x) \widetilde{g}(x)}{\mu(x)-\mu(c)} d x \\
& +\frac{4}{(d-c)^{2}} \int_{\frac{c+d}{2}}^{d} \frac{\rho(\mu(d)-\mu(x)) \mu^{\prime}(x) g(x)}{\mu(d)-\mu(x)} d x .
\end{aligned}
$$


Applying (1.4) in (3.1) and (3.2), we have, respectively,

$$
\begin{aligned}
\int_{0}^{1} \Delta_{\rho, \mu}(r) g^{\prime}\left(\frac{r}{2} d+\frac{2-r}{2} c\right) d r= & \frac{2}{d-c} \Delta_{\rho, \mu}(1) g\left(\frac{c+d}{2}\right)-\frac{4}{(d-c)^{2}}{ }_{\mu}^{\rho} F_{\left(\frac{c+d}{2}\right)^{-}} g(c) \\
& -\frac{4}{(d-c)^{2}}{ }_{\mu}^{\rho} F_{\left(\frac{c+d}{2}\right)}+\widetilde{g}(d),
\end{aligned}
$$

and

$$
\begin{aligned}
\int_{0}^{1} \Delta_{\rho, \mu}(r) g^{\prime}\left(\frac{r}{2} c+\frac{2-r}{2} d\right) d r= & -\frac{2}{d-c} \Delta_{\rho, \mu}(1) g\left(\frac{c+d}{2}\right)+\frac{4}{(d-c)^{2}}{ }_{\mu}^{\rho} F_{\left(\frac{c+d}{2}\right)^{-}} \widetilde{g}(c) \\
& +\frac{4}{(d-c)^{2}}{ }_{\mu}^{\rho} F_{\left(\frac{c+d}{2}\right)^{+}} g(d) .
\end{aligned}
$$

Equations (3.3) and (3.4) lead to required equality.

Remark 3.2. We can deduce associated identities for aforementioned fractional and conformable integrals from Lemma 3.1.

(i) If $\mu$ is the identity function, then we have [3, Lemma 3].

(ii) If $\rho(u)=\frac{u^{\frac{\gamma}{k}}}{k \Gamma_{k}(\gamma)}$, then we have,

$$
\begin{aligned}
& A_{1}^{\gamma, k}(1) g\left(\frac{c+d}{2}\right)-\frac{\Gamma_{k}(\gamma+k)}{2}\left[\underset{\mu}{\gamma} I_{\left(\frac{c+d}{2}\right)^{+}}^{k} G(d)+{ }_{\mu}^{\gamma} I_{\left(\frac{c+d}{2}\right)^{-}}^{k} G(c)\right] \\
& =\frac{d-c}{4} \int_{0}^{1} A_{1}^{\gamma, k}(\xi)\left(g^{\prime}\left(\frac{r}{2} d+\frac{2-r}{2} c\right)-g^{\prime}\left(\frac{r}{2} c+\frac{2-r}{2} d\right)\right) d r,
\end{aligned}
$$

where

$$
A_{1}^{\gamma, k}(\xi)=\left[\mu\left(\frac{r}{2} d+\frac{2-r}{2} c\right)-\mu(c)\right]^{\frac{\gamma}{k}}+\left[\mu(d)-\mu\left(\frac{r}{2} c+\frac{2-r}{2} d\right)\right]^{\frac{\gamma}{k}} .
$$

Moreover, if $k=1$, then it coincides to [21, Corollary 4].

(iii) If $\rho(u)=u^{\frac{\gamma}{k}} F_{\rho, \gamma}^{\sigma, k}\left(w(x)^{\rho}\right)$, then we get [21, Lemma 2]. Furthermore, if $k=1$, then we obtain [21, Corollary 3].

(iv) If we choose $\rho(u)=u^{\gamma} E_{\beta, \gamma, v}^{\delta, r, s, c}\left(w(u)^{\beta} ; q^{*}\right)$, then we obtain Lemma 2.1.

(v) If $\rho(u)=\frac{u}{\gamma} \exp (-A u)$, where $A=\frac{1-\gamma}{\gamma}, \gamma \in(0,1)$, then we have Lemma 2.2.

Theorem 3.3. In addition to the conditions of Lemma 3.1, if $\left|\mathrm{g}^{\prime}\right|$ is convex on $[\mathrm{c}, \mathrm{d}]$, then the following inequality for the generalized integral operator (1.4) holds:

$$
\left|\Delta_{\rho, \mu}(1) g\left(\frac{c+d}{2}\right)-\frac{1}{d-c}\left[\rho_{\mu}^{\rho} F_{\left(\frac{c+d}{2}\right)^{+}} G(d)+{ }_{\mu}^{\rho} F_{\left(\frac{c+d}{2}\right)^{-}} G(d)\right]\right| \leqslant \frac{(d-c)\left[\left|g^{\prime}(c)\right|+\left|g^{\prime}(d)\right|\right]}{4} \int_{0}^{1}\left|\Delta_{\rho, \mu}(r)\right| d r .
$$

Proof. From Lemma 3.1, we have

$$
\begin{aligned}
& \left|\Delta_{\rho, \mu}(1) g\left(\frac{c+d}{2}\right)-\frac{1}{d-c}\left[\rho_{\mu} F_{\left(\frac{c+d}{2}\right)}+G(d)+{ }_{\mu}^{\rho} F_{\left(\frac{c+d}{2}\right)}-G(d)\right]\right| \\
& \leqslant \frac{(d-c)}{4} \int_{0}^{1}\left|\Delta_{\rho, \mu}(r)\right|\left[\left|g^{\prime}\left(\frac{r}{2} d+\frac{2-r}{2} c\right)\right|+\left|g^{\prime}\left(\frac{r}{2} c+\frac{2-r}{2} d\right)\right|\right] d r .
\end{aligned}
$$

Now utilizing (2.10) in (3.5), we get the required inequality. 
Remark 3.4. We can deduce associated inequalities for various fractional and conformable integrals from Theorem 3.3.

(i) If $\mu$ is identity function, then we have [3, Theorem 4].

(ii) If $\rho(u)=\frac{u \frac{\gamma}{k}}{k \Gamma_{k}(\gamma)}$, then we have,

$$
\begin{aligned}
& \left|A_{1}^{\gamma, k}(1) g\left(\frac{c+d}{2}\right)-\frac{\Gamma_{k}(\gamma+k)}{2}\left[\underset{\mu}{\gamma} I_{\left(\frac{c+d}{2}\right)^{+}}^{k} G(d)+{ }_{\mu}^{\gamma} I_{\left(\frac{c+d}{2}\right)^{-}}^{k} G(c)\right]\right| \\
& \leqslant \frac{(d-c)\left[\left|g^{\prime}(c)\right|+\left|g^{\prime}(d)\right|\right]}{4} \int_{0}^{1}\left|A_{1}^{\gamma, k}(r)\right| d r
\end{aligned}
$$

where $A_{1}^{\gamma, k}(r)$ is same as in Remark 3.2. Moreover, if $k=1$, then it coincides to [21, Corollary 5].

(iii) If $\rho(u)=u^{\frac{\gamma}{k}} F_{\rho, \gamma}^{\sigma, k}\left(w(x)^{\rho}\right)$, then we obtain [21, Theorem 3].

(iv) If we choose $\rho(u)=u^{\gamma} E_{\beta, \gamma, r}^{\delta, r, s, c}\left(w(u)^{\beta} ; q^{*}\right)$, then we get Theorem 2.5.

(v) If $\rho(u)=\frac{u}{\gamma} \exp (-A u)$, where $A=\frac{1-\gamma}{\gamma}, \gamma \in(0,1)$, then we have Theorem 2.6.

Theorem 3.5. In addition to the conditions of Lemma 3.1, if $\left|\mathrm{g}^{\prime}\right|^{\mathrm{q}}, \mathrm{q}>1$ is convex on $[\mathrm{c}, \mathrm{d}]$, then the following inequality for the generalized integral operator (1.4) holds:

$$
\begin{aligned}
& \mid \Delta_{\rho, \mu}(1) g\left(\frac{\mathrm{c}+\mathrm{d}}{2}\right)-\frac{1}{\mathrm{~d}-\mathrm{c}}\left[\underset{\mu}{\rho} \mathrm{F}_{\left(\frac{\mathrm{c}+\mathrm{d}}{2}\right)^{+} \mathrm{G}(\mathrm{d})+_{\mu}^{\rho} \mathrm{F}_{\left.\left(\frac{\mathrm{c}+\mathrm{d}}{2}\right)^{-G}(\mathrm{~d})\right] \mid}}\right. \\
& \quad \leqslant \frac{(\mathrm{d}-\mathrm{c})}{4}\left(\int_{0}^{1}\left|\Delta_{\rho, \mu}(\mathrm{r})\right|^{\mathrm{p}} \mathrm{d} \xi\right)^{\frac{1}{p}}\left[\left(\frac{\left|g^{\prime}(\mathrm{d})\right|^{\mathrm{q}}+3\left|\mathrm{~g}^{\prime}(\mathrm{c})\right|^{\mathrm{q}}}{4}\right)^{\frac{1}{q}}+\left(\frac{\left|g^{\prime}(\mathrm{c})\right|^{\mathrm{q}}+3\left|\mathrm{~g}^{\prime}(\mathrm{d})\right|^{\mathrm{q}}}{4}\right)^{\frac{1}{q}}\right],
\end{aligned}
$$

where $\frac{1}{\mathrm{p}}+\frac{1}{\mathrm{q}}=1$.

Proof. By using the property of modulus in Lemma 3.1, we have

$$
\begin{aligned}
& \left|\Delta_{\rho, \mu}(1) g\left(\frac{\mathrm{c}+\mathrm{d}}{2}\right)-\frac{1}{\mathrm{~d}-\mathrm{c}}\left[{ }_{\mu}^{\rho} \mathrm{F}_{\left(\frac{\mathrm{c}+\mathrm{d}}{2}\right)}+\mathrm{G}(\mathrm{d})+{ }_{\mu}^{\rho} \mathrm{F}_{\left(\frac{\mathrm{c}+\mathrm{d}}{2}\right)^{-G}(\mathrm{c})}\right]\right| \\
& \quad \leqslant \frac{(\mathrm{d}-\mathrm{c})}{4}\left\{\int_{0}^{1}\left|\Delta_{\rho, \mu}(\mathrm{r})\right|\left|\mathrm{g}^{\prime}\left(\frac{\mathrm{r}}{2} \mathrm{~d}+\frac{2-\mathrm{r}}{2} \mathrm{c}\right)\right| \mathrm{d} r+\int_{0}^{1}\left|\Delta_{\rho, \mu}(\mathrm{r})\right|\left|g^{\prime}\left(\frac{\mathrm{r}}{2} \mathrm{c}+\frac{2-\mathrm{r}}{2} \mathrm{~d}\right)\right| \mathrm{dr}\right\} .
\end{aligned}
$$

By applying the Hölder's inequality, we get

$$
\int_{0}^{1}\left|\Delta_{\rho, \mu}(r)\right|\left|g^{\prime}\left(\frac{r}{2} d+\frac{2-r}{2} c\right)\right| d r \leqslant\left(\int_{0}^{1}\left|\Delta_{\rho, \mu}(r)\right|^{p} d r\right)^{\frac{1}{p}}\left(\int_{0}^{1}\left|g^{\prime}\left(\frac{r}{2} d+\frac{2-r}{2} c\right)\right|^{q} d r\right)^{\frac{1}{q}} .
$$

and

$$
\int_{0}^{1}\left|\Delta_{\rho, \mu}(r)\right|\left|g^{\prime}\left(\frac{r}{2} c+\frac{2-r}{2} d\right)\right| d r \leqslant\left(\int_{0}^{1}\left|\Delta_{\rho, \mu}(r)\right|^{p} d r\right)^{\frac{1}{p}}\left(\int_{0}^{1}\left|g^{\prime}\left(\frac{r}{2} c+\frac{2-r}{2} d\right)\right|^{q} d r\right)^{\frac{1}{q}} .
$$

Inequality (3.6) together with (2.14), (2.15), (3.7), and (3.8) leads to the required inequality. 
Remark 3.6. We deduce some inequalities for aforementioned fractional and conformable integrals from Theorem 3.5.

(i) If $\mu$ is identity function, then we have [3, Theorem 5].

(ii) If $\rho(u)=\frac{u \frac{\gamma}{k}}{k \Gamma_{k}(\gamma)}$, then we obtain,

$$
\begin{aligned}
& \left|A_{1}^{\gamma, k}(1) g\left(\frac{c+d}{2}\right)-\frac{\Gamma_{k}(\gamma+k)}{2}\left[\underset{\mu}{\gamma} \mathrm{I}_{\left(\frac{c+d}{2}\right)^{+}}^{k} \mathrm{G}(\mathrm{d})+{ }_{\mu}^{\gamma} \mathrm{I}_{\left(\frac{c+d}{2}\right)^{k}}^{\mathrm{k}} \mathrm{G}(\mathrm{c})\right]\right| \\
& \leqslant \frac{(\mathrm{d}-\mathrm{c})}{4}\left(\int_{0}^{1}\left|A_{1}^{\gamma, \mathrm{k}}(\mathrm{r})\right|^{\mathrm{p}} \mathrm{d} \xi\right)^{\frac{1}{\mathfrak{p}}}\left[\left(\frac{\left|g^{\prime}(\mathrm{d})\right|^{\mathrm{q}}+3\left|\mathrm{~g}^{\prime}(\mathrm{c})\right|^{\mathrm{q}}}{4}\right)^{\frac{1}{q}}+\left(\frac{\left|\mathrm{g}^{\prime}(\mathrm{c})\right|^{\mathrm{q}}+3\left|\mathrm{~g}^{\prime}(\mathrm{d})\right|^{\mathrm{q}}}{4}\right)^{\frac{1}{q}}\right],
\end{aligned}
$$

where $A_{1}^{\alpha, k}(r)$ is same as in Remark 3.2. Moreover, if $k=1$, then it will gives [21, Corollary 6].

(iii) If $\rho(u)=u^{\frac{\gamma}{k}} F_{\rho, \gamma}^{\sigma, k}\left(w(x)^{\rho}\right)$, then we get [21, Theorem 4].

(iv) If we choose $\rho(u)=u^{\gamma} E_{\beta, \gamma, v}^{\delta, r, s, c}\left(w(u)^{\beta} ; q^{*}\right)$, then we obtain Theorem 2.5.

(v) If $\rho(u)=\frac{u}{\gamma} \exp (-A u)$, where $A=\frac{1-\gamma}{\gamma}, \gamma \in(0,1)$, then we have Theorem 2.6.

\section{Applications}

Density functions and distribution functions give complete descriptions of the distribution of probability for a given random variable. However, they do not permit us to make comparisons between two different distributions easily. The set of moments are useful in making comparison under reasonable conditions. Recently, some researchers obtained error estimates for the moments of random variables by using mathematical inequalities, see for example $[1,2,4,11,12,17]$. Let $X$ be a random variable whose probability function is $\psi: I \subset \mathbb{R} \rightarrow \mathbb{R}^{+}$. The $\mathrm{m}^{\text {th }}$ moment about any arbitrary point $x$ of the random variable $X$ is denoted and defined as follows:

$$
M^{m}(x)=\int_{c}^{d}(t-x)^{m} \psi(t) d t, \quad m=0,1,2, \ldots
$$

Now we give some applications of our results for central moment. Now and onward we will use $\widehat{M^{m}}(u):=M^{m}(u)+M^{m}(c+d-u)$ for all $u \in[c, d]$ and $m \geqslant 2 s+1, s=0,1,2,3, \ldots$

Proposition 4.1. Let $X$ be a random variable whose probability function is $\psi: I \subset \mathbb{R} \rightarrow \mathbb{R}^{+}$, where $\psi$ is a convex function on the interval of real numbers I such that $\mathrm{c}, \mathrm{d} \in \mathrm{I}$ with $\mathrm{c}<\mathrm{d}$, then the following identity holds for the generalized integral operator (1.4):

$$
\begin{aligned}
& \Delta_{\rho, \mu}(1) M^{m}\left(\frac{c+d}{2}\right)-\frac{1}{d-c}\left[{ }_{\mu}^{\rho} F_{\left(\frac{c+d}{2}\right)^{-}} \widehat{M^{m}}(c)+{ }_{\mu}^{\rho} F_{\left(\frac{c+d}{2}\right)^{+}} \widehat{M^{m}}(d)\right] \\
& =\frac{m(d-c)}{4} \int_{0}^{1} \Delta_{\rho, \mu}(r)\left[M^{m-1}\left(\frac{r}{2} d+\frac{2-r}{2} c\right)-M^{m-1}\left(\frac{r}{2} c+\frac{2-r}{2} d\right)\right] d r .
\end{aligned}
$$

Proof. Required identity is obtained by setting $g(u)=M^{m}(u)$ in Lemma 3.1.

Remark 4.2. We can deduce associated identities for aforementioned fractional and conformable integrals from (4.1). 
(i) If $\rho(u)=\frac{u \frac{\gamma}{k}}{k \Gamma_{k}(\gamma)}$, then

$$
\begin{aligned}
& A_{1}^{\gamma, k}(1) M^{m}\left(\frac{c+d}{2}\right)-\frac{\Gamma_{k}(\gamma+k)}{2}\left[\underset{\mu}{\gamma} I_{\left(\frac{c+d}{2}\right)^{-}} \widehat{M^{m}}(c)+{ }_{\mu}^{\gamma} I_{\left(\frac{c+d}{2}\right)^{+}}^{k} \widehat{M^{m}}(d)\right] \\
& =\frac{m(d-c)}{4} \int_{0}^{1} A_{1}^{\gamma, k}(r)\left[M^{m-1}\left(\frac{r}{2} d+\frac{2-r}{2} c\right)-M^{m-1}\left(\frac{r}{2} c+\frac{2-r}{2} d\right)\right] d r
\end{aligned}
$$

where $A_{1}^{\gamma, k}(r)$ is same as in Remark 3.2.

(ii) If $\rho(u)=u^{\frac{\gamma}{k}} \mathcal{F}_{\rho, \gamma}^{\sigma, k}\left(w(u)^{\rho}\right)$, then

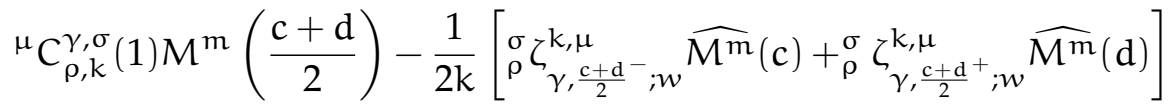

$$
\begin{aligned}
& =\frac{m(d-c)}{4} \int_{0}^{1} \mu C_{\rho, k}^{\gamma, \sigma}(r)\left[M^{m-1}\left(\frac{r}{2} d+\frac{2-r}{2} c\right)-M^{m-1}\left(\frac{r}{2} c+\frac{2-r}{2} d\right)\right] d r \text {, }
\end{aligned}
$$

where

$$
\begin{aligned}
{ }^{\mu} C_{\rho, k}^{\gamma, \sigma}(r)= & {\left[\mu(d)-\mu\left(\frac{r}{2} c+\frac{2-r}{2} d\right)\right]^{\frac{\gamma}{k}} \mathcal{F}_{\rho, \gamma+k}^{\sigma, k}\left(w\left[\mu(d)-\mu\left(\frac{r}{2} c+\frac{2-r}{2} d\right)\right]^{\rho}\right) } \\
& +\left[\mu\left(\frac{r}{2} d+\frac{2-r}{2} c\right)-\mu(c)\right]^{\frac{\gamma}{k}} \mathcal{F}_{\rho, \gamma+k}^{\sigma, k}\left(w\left[\mu\left(\frac{r}{2} d+\frac{2-r}{2} c\right)-\mu(c)\right]^{\rho}\right) .
\end{aligned}
$$

(iii) If we choose $\rho(u)=u^{\gamma} E_{\beta, \gamma, v}^{\delta, r, s, c}\left(w(u)^{\beta} ; q^{*}\right)$, then

$$
\begin{aligned}
& { }^{\mu} R_{\beta, \gamma, v}^{\delta, r, s, c}(1) M^{m}\left(\frac{c+d}{2}\right)-\frac{1}{d-c}\left[\underset{\mu}{w} \Upsilon_{\beta, \gamma, v, \frac{c+d}{2}}^{\delta, r, s, c}-\widehat{M^{m}}(c)+{ }_{\mu}^{w} \Upsilon_{\beta, \gamma, v, \frac{c+d}{2}}^{\delta, r, s, c} \widehat{M^{m}}(d)\right] \\
& =\frac{m(d-c)}{4} \int_{0}^{1}{ }^{\mu} R_{\beta, \gamma, v}^{\delta, r, s, c}(r)\left[M^{m-1}\left(\frac{r}{2} d+\frac{2-r}{2} c\right)-M^{m-1}\left(\frac{r}{2} c+\frac{2-r}{2} d\right)\right] d r .
\end{aligned}
$$

(iv) If $\rho(u)=\frac{u}{\gamma} \exp (-A u)$, where $A=\frac{1-\gamma}{\gamma}, \gamma \in(0,1)$, then

$$
\begin{aligned}
& N_{\gamma}^{\mu}(1) M^{m}\left(\frac{c+d}{2}\right)-\frac{1-\gamma}{2}\left[\underset{\mu}{\gamma} E_{\frac{c+d}{2}}-\widehat{M^{m}}(c)+{ }_{\mu}^{\gamma} E_{\frac{c+d}{2}}+\widehat{M^{m}}(d)\right] \\
& =\frac{m(d-c)}{4} \int_{0}^{1} N_{\gamma}^{\mu}(r)\left[M^{m-1}\left(\frac{r}{2} d+\frac{2-r}{2} c\right)-M^{m-1}\left(\frac{r}{2} c+\frac{2-r}{2} d\right)\right] .
\end{aligned}
$$

Proposition 4.3. Let $X$ be a random variable whose probability function is $\psi: I \subset \mathbb{R} \rightarrow \mathbb{R}^{+}$, where $\psi$ is a convex function on the interval of real numbers I such that $\mathrm{c}, \mathrm{d} \in \mathrm{I}$ with $\mathrm{c}<\mathrm{d}$. If $|\psi|$ is bounded, then the following inequality holds for the generalized integral operator (1.4):

$$
\begin{aligned}
& \left|\Delta_{\rho, \mu}(1) M^{m}\left(\frac{c+d}{2}\right)-\frac{1}{d-c}\left[\rho_{\mu} F_{\left(\frac{c+d}{2}\right)^{-}} \widehat{M^{m}}(c)+{ }_{\mu}^{\rho} F_{\left(\frac{c+d}{2}\right)^{+}} \widehat{M^{m}}(d)\right]\right| \\
& \leqslant \frac{\left(1-(-1)^{m}\right)(d-c)^{m+1}\|\psi\|_{\infty}}{4} \int_{0}^{1}\left|\Delta_{\rho, \mu}(r)\right|\left[\left(\frac{r}{2}\right)^{m}+\left(\frac{2-r}{2}\right)^{m}\right] d r .
\end{aligned}
$$


Proof. From Proposition 4.1, we have

$$
\begin{aligned}
& \left|\Delta_{\rho, \mu}(1) M^{m}\left(\frac{c+d}{2}\right)-\frac{1}{d-c}\left[{ }_{\mu}^{\rho} F_{\left(\frac{c+d}{2}\right)^{-}} \widehat{M^{m}}(c)+{ }_{\mu}^{\rho} F_{\left(\frac{c+d}{2}\right)^{+}} \widehat{M^{m}}(d)\right]\right| \\
& \leqslant \frac{m(d-c)}{4} \int_{0}^{1}\left|\Delta_{\rho, \mu}(r)\right|\left[\left|M^{m-1}\left(\frac{r}{2} d+\frac{2-r}{2} c\right)\right|+\left|M^{m-1}\left(\frac{r}{2} c+\frac{2-r}{2} d\right)\right|\right] d r .
\end{aligned}
$$

The required estimate is obtained by evalauting the integrals on the right side of the inequality.

Remark 4.4. We now deduce new inequalities involving central moment of a random variable for different fractional and conformable integrals from Proposition 4.3.

(i) If $\rho(u)=\frac{u \frac{\gamma}{k}}{k \Gamma_{k}(\gamma)}$, then

$$
\begin{aligned}
& \left|A_{1}^{\gamma, k}(1) M^{m}\left(\frac{c+d}{2}\right)-\frac{\Gamma_{k}(\gamma+k)}{2}\left[{ }_{\mu}^{\gamma} I_{\left(\frac{c+d}{2}\right)^{-}}^{k} \widehat{M^{m}}(c)+{ }_{\mu}^{\gamma} I_{\left(\frac{c+d}{2}\right)^{+}}^{k} \widehat{M^{m}}(d)\right]\right| \\
& \quad \leqslant \frac{m(d-c)}{4} \int_{0}^{1}\left|A_{1}^{\gamma, k}(r)\right|\left[\left|M^{m-1}\left(\frac{r}{2} d+\frac{2-r}{2} c\right)\right|+\left|M^{m-1}\left(\frac{r}{2} c+\frac{2-r}{2} d\right)\right|\right] d r,
\end{aligned}
$$

where $A_{1}^{\gamma, k}(r)$ is same as in Remark 3.2.

(ii) If $\rho(u)=u^{\frac{\gamma}{k}} \mathcal{F}_{\rho, \gamma}^{\sigma, k}\left(w(u)^{\rho}\right)$, then

$$
\begin{aligned}
& \left|{ }^{\mu} C_{\rho, k}^{\gamma, \sigma}(1) M^{m}\left(\frac{c+d}{2}\right)-\frac{1}{2 k}\left[{ }_{\rho}^{\sigma} \zeta_{\gamma, \frac{c+d}{2}-; w}^{k, \mu} \widehat{M^{m}}(c)+{ }_{\rho}^{\sigma} \zeta_{\gamma, \frac{c+d}{2}+;}^{k, \mu} \widehat{M^{m}}(d)\right]\right| \\
& \leqslant \frac{\left(1-(-1)^{m}\right)(d-c)^{m+1}\|\psi\|_{\infty}}{4} \int_{0}^{1}\left|\mu C_{\rho, k}^{\gamma, \sigma}(r)\right|\left[\left(\frac{r}{2}\right)^{m}+\left(\frac{2-r}{2}\right)^{m}\right] d r,
\end{aligned}
$$

where ${ }^{\mu} \mathrm{C}_{\rho, k}^{\gamma, \sigma}(r)$ is given in (4.2).

(iii) If we choose $\rho(u)=u^{\gamma} E_{\beta, \gamma, v}^{\delta, r, s, c}\left(w(u)^{\beta} ; q^{*}\right)$, then

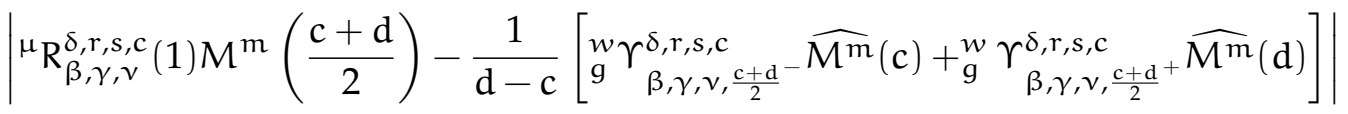

$$
\begin{aligned}
& \leqslant \frac{\left(1-(-1)^{m}\right)(d-c)^{m+1}\|\psi\|_{\infty}}{4} \int_{0}^{1}\left|\mu R_{\beta, \gamma, v}^{\delta, r, s, c}(r)\right|\left[\left(\frac{r}{2}\right)^{m}+\left(\frac{2-r}{2}\right)^{m}\right] d r .
\end{aligned}
$$

(iv) If $\rho(u)=\frac{u}{\gamma} \exp (-A u)$, where $A=\frac{1-\gamma}{\gamma}, \gamma \in(0,1)$, then

$$
\begin{aligned}
& \left|N_{\gamma}^{\mu}(1) M^{m}\left(\frac{c+d}{2}\right)-\frac{1-\gamma}{2}\left[\gamma_{\mu}^{\gamma} E_{\frac{c+d}{2}}-\widehat{M^{m}}(c)+\gamma_{\mu}^{\gamma} E_{\frac{c+d}{2}} \widehat{M^{m}}(d)\right]\right| \\
& \leqslant \frac{\left(1-(-1)^{m}\right)(d-c)^{m+1}\|\psi\|_{\infty}}{4} \int_{0}^{1}\left|N_{\gamma}^{\mu}(r)\right|\left[\left(\frac{r}{2}\right)^{m}+\left(\frac{2-r}{2}\right)^{m}\right] d r .
\end{aligned}
$$

Proposition 4.5. Let $X$ be a random variable whose probability function is $\psi: I \subset \mathbb{R} \rightarrow \mathbb{R}^{+}$, where $\psi$ is a convex function on the interval of real numbers I such that $\mathrm{c}, \mathrm{d} \in \mathrm{I}$ with $\mathrm{c}<\mathrm{d}$ and $\psi \in \mathrm{L}_{\mathrm{p}}[\mathrm{c}, \mathrm{d}], \mathrm{p}>1$. If $|\psi|$ is bounded, 
then

$$
\begin{aligned}
& \left|\Delta_{\rho, \mu}(1) M^{m}\left(\frac{c+d}{2}\right)-\frac{1}{d-c}\left[\rho_{\mu}^{\rho} F_{\left(\frac{c+d}{2}\right)^{-}} \widehat{M^{m}}(c)+{ }_{\mu}^{\rho} F_{\left(\frac{c+d}{2}\right)^{+}} \widehat{M^{m}}(d)\right]\right| \\
& \leqslant \frac{m\left(1-(-1)^{(m-1) q+1}\right)(d-c)^{(m-1) q+2}\|\psi\|_{p}}{4((m-1) q+1)} \int_{0}^{1}\left|\Delta_{\rho, \mu}(r)\right|\left[\left(\frac{r}{2}\right)^{(m-1) q+1}+\left(\frac{2-r}{2}\right)^{(m-1) q+1}\right] d r .
\end{aligned}
$$

Proof. From Proposition 4.1, we get

$$
\begin{aligned}
& \left|\Delta_{\rho, \mu}(1) M^{m}\left(\frac{c+d}{2}\right)-\frac{1}{d-c}\left[{ }_{\mu}^{\rho} F_{\left(\frac{c+d}{2}\right)^{-}} \widehat{M^{m}}(c)+{ }_{\mu}^{\rho} F_{\left(\frac{c+d}{2}\right)^{+}} \widehat{M^{m}}(d)\right]\right| \\
& \leqslant \frac{m(d-c)}{4} \int_{0}^{1}\left|\Delta_{\rho, \mu}(r)\right|\left[\left|M^{m-1}\left(\frac{r}{2} d+\frac{2-r}{2} c\right)\right|+\left|M^{m-1}\left(\frac{r}{2} c+\frac{2-r}{2} d\right)\right|\right] d r .
\end{aligned}
$$

By the Hölder's inequality, we have

$$
\begin{gathered}
\left|M^{m-1}\left(\frac{r}{2} d+\frac{2-r}{2} c\right)\right| \leqslant \int_{c}^{d}\left(x-\left(\frac{r}{2} d+\frac{2-r}{2}\right)\right)^{m-1}|\psi(x)| d x \\
\leqslant\left(\int_{c}^{d}|\psi(x)|^{p} d x\right)^{\frac{1}{p}}\left(\int_{c}^{d}\left(x-\left(\frac{r}{2} d+\frac{2-r}{2}\right)\right)^{(m-1) q} d x\right)^{\frac{1}{q}} .
\end{gathered}
$$

Inequalities (4.3) and (4.4) give the required estimate.

Remark 4.6. We deduce associated inequalities from Proposition 4.5 for central moment of a random variable via different fractional and conformable integrals.

(i) If $\rho(u)=\frac{u \frac{\gamma}{k}}{k \Gamma_{k}(\gamma)}$, then

$$
\begin{aligned}
& \left|A_{1}^{\gamma, k}(1) M^{m}\left(\frac{c+d}{2}\right)-\frac{\Gamma_{k}(\gamma+k)}{2}\left[{ }_{\mu}^{\gamma} I_{\left(\frac{c+d}{2}\right)^{-}}^{k} \widehat{M^{m}}(c)+{ }_{\mu}^{\gamma} I_{\left(\frac{c+d}{2}\right)^{+}}^{k} \widehat{M^{m}}(d)\right]\right| \\
& \leqslant \frac{m\left(1-(-1)^{(m-1) q+1}\right)(d-c)^{(m-1) q+2}\|\psi\|_{p}}{4((m-1) q+1)} \int_{0}^{1}\left|A_{1}^{\gamma, k}(r)\right|\left[\left(\frac{r}{2}\right)^{(m-1) q+1}+\left(\frac{2-r}{2}\right)^{(m-1) q+1}\right] d r,
\end{aligned}
$$

where $A_{1}^{\alpha, k}(r)$ is same as in Remark 3.2.

(ii) If $\rho(u)=u^{\frac{\gamma}{k}} \mathcal{F}_{\rho, \gamma}^{\sigma, k}\left(w(u)^{\rho}\right)$, then

$$
\begin{aligned}
& \left|{ }^{\mu} C_{\rho, k}^{\gamma, \sigma}(1) M^{m}\left(\frac{c+d}{2}\right)-\frac{1}{2 k}\left[{ }_{\rho}^{\sigma} \zeta_{\gamma, \frac{c+d}{2}{ }^{k} ; w} \widehat{M^{m}}(c)+{ }_{\rho}^{\sigma} \zeta_{\gamma, \frac{c+d}{2}+;}^{k, \mu} \widehat{M^{m}}(d)\right]\right| \\
& \leqslant \frac{m\left(1-(-1)^{(m-1) q+1}\right)(d-c)^{(m-1) q+2}\|\psi\|_{p}}{4((m-1) q+1)} \int_{0}^{1}\left|\mu C_{\rho, k}^{\gamma, \sigma}(r)\right|\left[\left(\frac{r}{2}\right)^{(m-1) q+1}+\left(\frac{2-r}{2}\right)^{(m-1) q+1}\right] d r,
\end{aligned}
$$

where ${ }^{\mu} C_{\rho, k}^{\gamma, \sigma}(r)$ is same as in (4.2). 
(iii) If we choose $\rho(u)=u^{\gamma} E_{\beta, \gamma, v}^{\delta, r, s, c}\left(w(u)^{\beta} ; q^{*}\right)$, then

$$
\begin{aligned}
& \left|\mu_{R_{\beta, \gamma, \gamma}^{\delta, r, s, c}}^{\delta}(1) M^{m}\left(\frac{c+d}{2}\right)-\frac{1}{d-c}\left[\underset{g}{w} \Upsilon_{\beta, \gamma, v, \frac{c+d}{2}}^{\delta, r, s, c}-\widehat{M^{m}}(c)+{ }_{g}^{w} \Upsilon_{\beta, \gamma, v, \frac{c+d}{2}}^{\delta, r, s, c}+\widehat{M^{m}}(d)\right]\right| \\
& \leqslant \frac{m\left(1-(-1)^{(m-1) q+1}\right)(d-c)^{(m-1) q+2}\|\psi\|_{p}}{4((m-1) q+1)} \int_{0}^{1}\left|\mu R_{\beta, \gamma, v}^{\delta, r, s, c}(r)\right|\left[\left(\frac{r}{2}\right)^{(m-1) q+1}+\left(\frac{2-r}{2}\right)^{(m-1) q+1}\right] d r .
\end{aligned}
$$

(iv) If $\rho(u)=\frac{u}{\gamma} \exp (-A u)$, where $A=\frac{1-\gamma}{\gamma}, \gamma \in(0,1)$, then

$$
\begin{aligned}
& \left|N_{\gamma}^{\mu}(1) M^{m}\left(\frac{c+d}{2}\right)-\frac{1-\gamma}{2}\left[{ }_{\mu}^{\gamma} E_{d^{-}} \widehat{M^{m}}(c)+{ }_{\mu}^{\gamma} E_{d^{+}} \widehat{M^{m}}(d)\right]\right| \\
& \leqslant \frac{m\left(1-(-1)^{(m-1) q+1}\right)(d-c)^{(m-1) q+2}\|\psi\|_{p}}{4((m-1) q+1)} \int_{0}^{1}\left|N_{\gamma}^{\mu}(r)\right|\left[\left(\frac{r}{2}\right)^{(m-1) q+1}+\left(\frac{2-r}{2}\right)^{(m-1) q+1}\right] d r
\end{aligned}
$$

\section{Concluding remarks}

In this paper, we developed some generalized inequalities of mid-point type via generalized $k$-fractional integral and generalized exponential fractional integral for differentiable convex functions. We obtained some new generalized inequalities via the generalized integral operator and deduced some new inequalities for the various fractional integrals. We have applied our results to construct new inequalities for the moments of a continuous random variable. Lastly, we conclude that our results would provide generalizations of those given in previous works. We feel that some generalized inequalities can be obtained by using different kinds of convex functions via generalized integral operator (1.4). We hope that this work will attract the attention of the researchers working in fractional calculus, mathematical analysis, and other related fields.

\section{Acknowledgment}

We would like to express our gratitude to the Editor and anonymous referees for their valuable suggestions and comments that helped us in improving this manuscript.

\section{References}

[1] N. S. Barnett, P. Cerone, S. S. Dragomir, J. Roumeliotis, Some inequalities for the dispersion of a random variable whose pdf is defined on a finite interval, J. Inequal. Pure Appl. Math., 2 (2001), 18 pages. 4

[2] N. S. Barnett, S. S. Dragomir, Some elementary inequalities for the expectation and variance of a random variable whose pdf is defined on a finite interval, Nova Sci. Publ., 2 (2002), 31-38. 4

[3] H. Budak, F. Ertugral, M. Z. Sarikaya, New generalization of Hermite-Hadamard type inequalities via generalized fractional integrals. i, i, i

[4] P. Cerone, S. S. Dragomir, On some inequalities for the expectation and variance, Korean J. Comput. Appl. Math., 8 (2001), 357-380. 4

[5] S. S. Dragomir, Inequalities of Jensen's type for generalized k-g-Fractional integrals of functions for which the composite fo $\mathrm{g}^{-1}$ is convex, Fract. Differ. Calc., 8 (2018), 127-150. 1

[6] G. Farid, Existence of an integral operator and its consequences in fractional calculus, Open J. Math. Sci., 3 (2019), 210-216. 1

[7] G. Farid, M. Raees, M. Anwar, Bounds associated to Hadamard inequality via generalized integral operators and applications for conformable and fractional integrals, J. Fract. Calc. Appl., 11 (2020), 238-251. 1

[8] A. Fernandez, P. O. Mohammed, Hermite-Hadamard inequalities in fractional calculus defined using Mittag-Leffler kernels, Math. Methods Appl. Sci., 2020 (2020), 18 pages. 1

[9] S. M. Kang, G. Farid, W. Nazeer, B. Tariq, Hadamard and Fejer-Hadamard inequalities for extended generalized fractional integrals involving special functions, J. Inequal. Appl., 2018 (2018), 11 pages. 1, 1, 1 
[10] A. A. Kilbas, H. M. Srivastava, J. J. Trujillo, Theory and applications of fractional differential equations, Elsevier Science B.V., Amsterdam, (2006). 1

[11] P. Kumar, Moments inequalities of a random variable defined over a finite interval, JIPAM. J. Inequal. Pure Appl. Math., 3 (2002), 11 pages. 4

[12] P. Kumar, Inequalities involving moments of a continuous random variable defined over a finite interval, Comput. Math. Appl., 48 (2004), 257-273. 4

[13] Y. C. Kwun, G. Farid, W, Nazeer, S. Ullah, S. M. Kang, Generalized Riemann-Liouville k-fractional integrals associated with Ostrowski type inequalities and error bounds of Hadamard Inequalities, IEEE Access, 6 (2018), 64946-64953. 1

[14] P. O. Mohammed, Hermite-Hadamard inequalities for Riemann-Liouville fractional integrals of a convex function with respect to a monotone function, Math. Meth. Appl. Sci., 2019 (2019), 11 pages. 1

[15] P. O. Mohammed, T. Abdeljawad, Modification of certain fractional integral inequalities for convex functions, Adv. Difference Equ., 2020 (2020), 22 pages.

[16] P. O. Mohammed, I. Brevik, A New Version of the Hermite-Hadamard Inequality for Riemann-Liouville Fractional Integrals, Symmetry, 12 (2020), 11 pages. 1

[17] P. O. Mohammad, M. Z. Sarikaya, On generalized fractional integral inequalities for twice differentiable convex functions, J. Comput. Appl. Math., 372 (2020), 15 pages. 4

[18] P. O. Mohammed, M. Z. Sarikaya, D. Baleanu, On the Generalized Hermite-Hadamard Inequalities via the Tempered Fractional Integrals, Symmetry, 12 (2020), 17 pages. 1

[19] S. G. Samko, A. A. Kilbas, O. I. Marichev, Fractional Integrals and Derivatives, Gordon and Breach Science Publishers, Yverdon, (1993). 1

[20] M. Z. Sarikaya, E. Set, H. Yaldiz, N. Başak, Hermite-Hadamard's inequlities for fractional integrals and related fractional inequalitis, Math. Comput. Modelling, 57 (2013), 2403-2407. 1

[21] E. Set, J. S. Choi, A. Gözpinar, Hermite-Hadamard type inequalities for the generalized k-fractional integral operators, J. Inequal. Appl., 2017 (2017), 17 pages. 1, 1, ii, iii, ii, iii, ii, iii

[22] T. Tunc, H. Budak, F. Usta, M.Z. Sarikaya, On new generalized fractional integral operators and related fractional inequalities. 1 